3 MEARS, R. J., REEKIE, L., POOLE, S. B., and PAYNE, D. N.: 'Lowthreshold tunable $\mathrm{CW}$ and $Q$-switched fibre laser operating at $1 \cdot 55 \mu \mathrm{m}$ ', Electron. Lett., 1986, 22, pp. 159-160

4 JAUNCEY, I. M., REEKIE, L., MEARS, R. J., and ROWE, C. J.: 'Narrow linewidth fibre laser operating at $1.55 \mu \mathrm{m}$ ', Opt. Lett., 1987, 12, pp. $164-165$

5 MEARS, R. J., REEKIE, L., JAUNCEY, I. M., and PAYNE, D. N.: 'High-gain rare-earth doped fibre amplifier at $1.54 \mu \mathrm{m}$ '. OFC, Reno, USA, 1987

6 MILlaR, C. A., MILLER, I. D., AINSLIE, B. J., CRAIG, S. P., and ARMITAGE, J. R.: 'Low-threshold CW operation of an erbium-doped fibre laser pumped at $807 \mathrm{~nm}$ wavelength', Electron. Lett., 1987, 23, pp. 865-866

7 REEKIE, L., MEARS, R. J., POOLE, S. B., and PAYNE, D. N.: 'Optical bistability at $1.55 \mu \mathrm{m}$ in an $\mathrm{Er}^{3+}$-doped single-mode fibre laser'. ECOC '86, Barcelona, Spain, 1986

8 TOWNSEND, J. E., POOLE, S. B., and PAYNE, D. N.: 'Solution doping technique for fabrication of rare-earth-doped optical fibres', Electron. Lett., 1987, 23, pp. 329-331

\section{MODULATION FREQUENCY-SHIFT TECHNIQUE FOR DISPERSION MEASUREMENTS IN OPTICAL FIBRES USING LEDS}

Indexing terms: Optical fibres, Optical measurement, LEDs, Optical dispersion

Reliable phase-shift measurements in optical fibres were performed by downshifting the modulation frequency of the optical signal to the low-frequency range before detection. This technique maintains the full resolution of the highfrequency modulation and increases the sensitivity allowing accurate group delay measurements in single-mode fibres over a $300 \mathrm{~nm}$ spectral range using a single LED.

Introduction: The measurement of chromatic dispersion of single-mode fibres is of greatest importance to ensure optimal performance in the data rate capability of an optical link. Many experimental methods have been proposed to date, ${ }^{1}$ and among them phase-shift techniques using LEDs are predominant. $^{2}$ The main problem arising with this technique is the low intensity of the detected light, resulting in a poor signal/noise ratio and strong phase fluctuations. Furthermore, radio-frequency interferences due to the strong LED drive current perturb the measured signal, providing biased measurements. These problems can be reduced only by using extreme care and advanced techniques. ${ }^{3}$

In this letter we report a technique giving improved phaseshift measurements using optical signal processing. This novel method combines the advantages of good resolution due to high-frequency modulation and the extreme sensitivity and reliability due to low-frequency detection and signal processing. The method fully eliminates the main problems encountered with phase-shift techniques, even improving the performance.

Basic principles: When the intensity of an optical source is sinusoidally modulated and its light is launched into a singlemode fibre, the output signal detected is

$$
I=I_{0}(\lambda) \cos \left(\omega_{1} t+\phi_{1}+\tau(\lambda) \omega_{1} L\right)
$$

where $I_{0}(\lambda)$ is the output optical modulation amplitude (depending on the LED spectrum and fibre attentuation), $\omega_{1}$ the modulation angular frequency, $\phi_{1}$ an arbitrary constant phase, $\tau(\lambda)$ the group delay at the emission wavelength $\lambda$, and $L$ the fibre length. Thus the measurement of the modulation phase straightforwardly yields the relative group delay as a function of wavelength, providing that the source is wavelength-tunable. As it is a scaling factor in the phase expression, the modulation frequency must be high enough for good resolution, and is actually greater than $10 \mathrm{MHz}$. Direct phase measurement at the modulation frequency is therefore possible only with a relatively high-speed optical receiver with consequently high noise level.

When a second intensity modulation at an angular frequency $\omega_{2}$ is optically performed between the fibre output and the detector, the detected signal becomes

$$
\begin{aligned}
I= & I_{0}(\lambda) m_{2} \cos \left(\omega_{1} t+\phi_{1}+\tau(\lambda) \omega_{1} L\right) \\
& \times \cos \left(\omega_{2} t+\phi_{2}\right) \\
= & \frac{1}{2} I_{0}(\lambda) m_{2}\left\{\operatorname { c o s } \left[\left(\omega_{1}-\omega_{2}\right) t+\phi_{1}-\phi_{2}\right.\right. \\
& \left.+\tau(\lambda) \omega_{1} L\right]+\cos \left[\left(\omega_{1}+\omega_{2}\right) t+\phi_{1}+\phi_{2}\right. \\
& \left.\left.+\tau(\lambda) \omega_{1} L\right]\right\}
\end{aligned}
$$

where $m_{2}$ is the second modulation depth and $\phi_{2}$ is another arbitrary constant phase. Thus this second modulation generates sum- and difference-frequency signals, each conserving the phase information of the primary signal. The second modulation frequency $\omega_{2}$ may be chosen very close to $\omega_{1}$ so that the difference-frequency component lies in the $0 \cdot 1-1 \mathrm{kHz}$ range, where ultrasensitive optical detection and highresolution phase measurements can be performed. Therefore this frequency transformation enables performance of lowfrequency measurements of phase shifts due to high-frequency modulation.

Experimental description: A schematic diagram of the experimental set-up is shown in Fig. 1. Both modulation frequencies

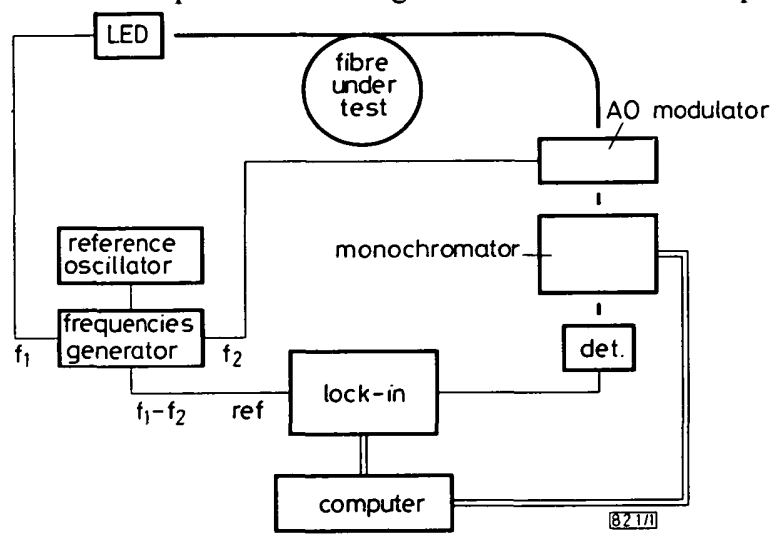

Fig. 1 Schematic diagram of experimental set-up

and their difference are electronically generated by the reference oscillator. One of these signals directly modulates the current driving an RCA C86013E edge-emitting LED with nominal centre wavelength at $1280 \mathrm{~nm}$. Emitted light is launched into the single-mode fibre under test. At the receiving end the second modulation is achieved by using a dopedglass acousto-optic modulator enabling a $60 \%$ modulation depth. The output from the modulator is directed through a single-mode fibre to a monochromator which performs wavelength scanning and filtering with a $5 \mathrm{~nm}$ FWHM. A GaInAsP PIN photodiode operating in the photovoltaic mode generates the electrical signal detected by a two-phase lock-in amplifier. The reference signal is the difference frequency provided by the frequency generator. The intrinsically high stability and strong filtering capability of the lock-in amplifier enable measurements with a $0.1 \mathrm{deg}$ accuracy, fully independent of the signal amplitude.

Results: With this set-up the level of RF interference was not measurable and the noise contribution can be arbitrarily reduced by lock-in time integration, so that the dynamic range is finally determined by a reasonable measurement duration. Spectral coverage is limited by the optical attenuation of the fibre under test owing to the rapidly decreasing intensity in the tails of the LED emission spectrum. With our set-up an overall attenuation of $30 \mathrm{~dB}$ allows $100 \mathrm{~nm}$ spectral coverage. If the overall attenuation can be reduced to $10 \mathrm{~dB}$ the spectral coverage increases to $200 \mathrm{~nm}$, and can be extended to $300 \mathrm{~nm}$ if the attenuation is only $2 \mathrm{~dB}$. Fig. 2 shows group delay/ wavelength results of a $3.7 \mathrm{~km}$ single-mode fibre manufactured by Cabloptic. This step-index fibre has a maximum attenu- 
ation of $0.75 \mathrm{~dB} / \mathrm{km}$ at the $\mathrm{OH}^{-}$attenuation peak. This low maximum attenuation enabled measurements to be made from $1130 \mathrm{~nm}$ to $1450 \mathrm{~nm}$ with a lock-in integration time of less than $3 \mathrm{~s}$.

The group delay accuracy is limited by the LED modulation frequency, noise level and lock-in phase resolution. The noise contribution can be sufficiently reduced by an appropriate choice of integration time so that phase accuracy is given

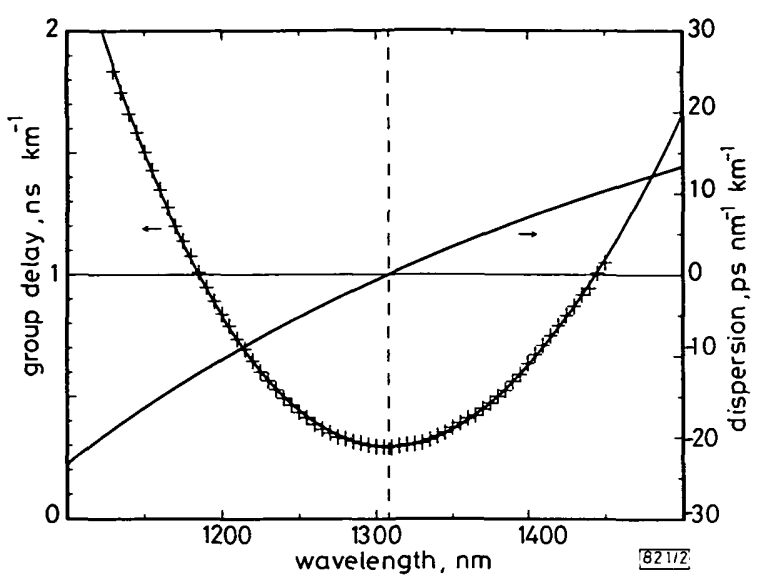

Fig. 2 Measured group delays of a $3.7 \mathrm{~km}$ single-mode fibre using a single LED and fitted Sellmeier polynomial, with resulting chromatic dispersion curve

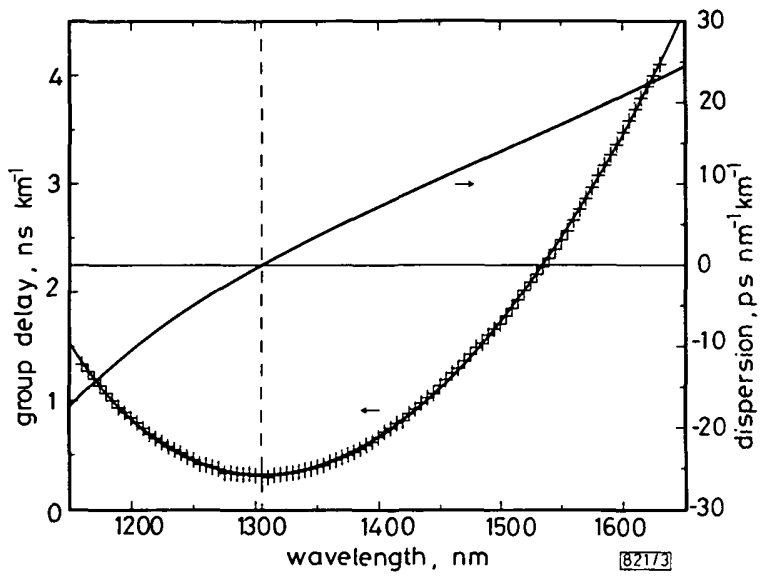

Fig. 3 Measured group delays of a $3.7 \mathrm{~km}$ single-mode fibre using coupled outputs of two LEDs and fitted Sellmeier polynomial, with resulting chromatic dispersion curve by the $0 \cdot 1 \mathrm{deg}$ apparatus resolution. The equivalent group delay resolution is about $6 \mathrm{ps}$ with a $50 \mathrm{MHz}$ LED modulation frequency in our case. The measurement of 12 independent group delay spectra gave a standard deviation of $0.47 \mathrm{~nm}$ for the zero chromatic dispersion wavelength.

For continuous measurements in both communication transmission windows, a wide spectral coverage set-up was achieved using a single-mode coupler merging the light outputs of two LEDs having nominal centre wavelengths of $1280 \mathrm{~nm}$ and $1500 \mathrm{~nm}$, respectively. In this case special care must be taken to equalise the modulation phases of the LEDs to obtain smooth phase shifts where the sources' spectra overlap. A measurement in such a configuration is shown in Fig. 3 for the same $3.7 \mathrm{~km}$ fibre.

Conclusion: This modulation frequency-shift technique for group delay measurements by phase shifts uses the advantages of a low-frequency detection scheme and nevertheless keeps the resolution of high-frequency modulation. Reliability and performance are improved with respect to other methods owing to the extreme sensitivity of detection and the excellent stability of the signal processing.

Acknowledgments: We are grateful to F. Sandoz of Cabloptic for kindly supplying the fibre, and to K. Jauch of Alphatronix for helpful comments.

\section{THEVENAZ}

30th July 1987

Group of Applied Physics

University of Geneva

20 Rue de l'Ecole-de-Medecine

CH-1211 Geneva 4, Switzerland

\section{J.-P. PELlaUX}

Alphatronix $S A$

111 Chemin du Pont-du-Centenaire

CH-1228 Plan-les-Ouates, Switzerland

\section{References}

1 COHEN, L. G., and LIN, C.: 'Comparison of single-mode fiber dispersion measurement techniques', J. Lightwave Technol., 1985, LT-3, pp. $958-966$

2 COSTA, B., PUleo, M., and vezzonl, E.: 'Phase-shift technique for the measurement of chromatic dispersion in single-mode optical fibres using LEDs', Electron. Lett., 1983, 19, pp. 1074-1076

3 SLADEN, F. M. E., REICHARD, H. S., and UVEGESS, s.: 'Chromatic dispersion measurements on long fibre lengths using LEDs', ibid., 1986, 22, pp. 841-842

\section{MOLECULAR-BEAM-EPITAXIAL GROWTH OF GaAs ON HIGH-TEMPERATURE HYDROGEN-ANNEALED (100) SILICON}

\author{
Indexing terms: Semiconductor devices and materials, Epi- \\ taxial growth, GaAs, Silicon
}

Superior-quality GaAs epitaxial layers have been grown by molecular beam epitaxy on high-temperature hydrogenambient annealed silicon (100) substrates. Rutherford backscattering and channelling of $2 \cdot 1 \mathrm{MeV} \mathrm{He}^{+}$ions and transmission electron microscopy techniques have been used to characterise these layers. Comparative studies indicate that the epitaxial layers grown on hydrogen-ambient annealed substrates have a superior surface morphology and a lower interface disorder than those on chemically cleaned (100) substrates. Cross-sectional transmission electron micrographs show the presence of a high density of threading dislocations, stacking faults and twins in the GaAs layers grown on the chemically cleaned silicon (100) substrates. In contrast, a significant reduction in the density of these defects is observed in the layers grown on the preannealed substrates.

Heteroepitaxial GaAs on silicon is a very promising material for the fabrication of monolithic electronic and optoelectronic integrated circuits. ${ }^{1,2}$ To date, single-crystal GaAs epitaxial layers have been grown on silicon substrates with various crystallographic orientations, ${ }^{3,4}$ with or without intermediate buffered layers ${ }^{5}$ and including strained-layer superlatties. ${ }^{6}$ In this letter we report the growth of superior-quality GaAs epitaxial layers on high-temperature hydrogen-ambient annealed silicon (100) substrates.

Several samples were cut from a $\sim 4 \Omega \mathrm{cm} n$-type (100) silicon wafer and chemically cleaned by a technique originally developed by Kern and Puotinen at RCA, and hence, commonly referred to as the RCA cleaning technique. ${ }^{7}$ Following this procedure, a few samples were annealed in a hydrogenambient at a temperature of $1250^{\circ} \mathrm{C}$ for $30 \mathrm{~min}$. The chemically cleaned and the hydrogen-annealed samples were then mounted side-by-side on a molybdenum sample holder using an indium solder and transferred into the molecular beam epitaxy system. The native oxide was desorbed by heating the substrates to a temperature of $780^{\circ} \mathrm{C}$ for $20 \mathrm{~min}$. The substrate temperature was then lowered to $415^{\circ} \mathrm{C}$ and an As primer layer deposited. After the initial growth of a thin layer of GaAs, the growth temperature was increased to $600^{\circ} \mathrm{C}$. The deposition was carried out for $1 \mathrm{~h}$. The fluxes were calibrated for a growth rate of $1 \mu \mathrm{m} / \mathrm{h}$, established for homoepitaxial GaAs.

These samples and a homoepitaxial GaAs film were then analysed by Rutherford backscattering and channelling using $2 \cdot 1 \mathrm{MeV} \mathrm{He}{ }^{+}$ions. Good crystalline quality is indicated in both cases by observed low channelled yields. Determined values of $\chi$, the ratio of aligned to nonaligned yield, are given in Table 1 . The crystalline quality at the surface and the interface is evaluated in terms of $\chi_{\min }$ and $\chi_{i n t}$, respectively. Values 